\title{
'Universal' curve of ionic conductivities in binary alkali germanate glasses
}

\author{
Marcio Luis Ferreira Nascimento ${ }^{\mathrm{a}, *}$, Eduardo do Nascimento ${ }^{\mathrm{b}}$, Shigueo Watanabe ${ }^{\mathrm{b}}$ \\ ${ }^{a}$ Department of Materials Engineering, Federal University of São Carlos, Rod. Washington Luiz, km 235, São Carlos 13565-905, Brazil \\ ${ }^{\mathrm{b}}$ Institute of Physics, University of São Paulo, Rua do Matão 187, São Paulo 05508-900, Brazil
}

Received 9 February 2005; received in revised form 2 June 2005; accepted 19 June 2005

\begin{abstract}
An analysis and brief discussion of experimental ionic conductivity $\sigma$ in the binary alkali germanate system is presented, exemplified on 31 binary $\mathrm{GeO}_{2}$ glasses that span ionic conductivity by more than 17 orders of magnitude in a wide composition range. An 'universal' finding using modified Arrhenius plots $\log \sigma$ or $\log \sigma T$ versus $E_{\mathrm{A}} / k_{\mathrm{B}} T$ is obtained, where $E_{\mathrm{A}}$ is the activation enthalpy for conduction, $k_{\mathrm{B}}$ the Boltzmann constant and $T$ is the absolute temperature. For a given value of $E_{\mathrm{A}} / k_{\mathrm{B}} T$, the difference between large to small values of $\sigma$ is only about one order of magnitude in $87 \%$ of the glass systems considered. The fact that $\sigma$ lies on these single 'universal' curves for so many ion-conducting binary germanate glasses means that $\sigma$ is governed mainly by $E_{\mathrm{A}}$ while the pre-exponential factor $\sigma_{0}$ varies around an average value $\approx 50 \mathrm{~S} \mathrm{~cm}^{-1}$. The activation enthalpy composition dependence is explained concerning the Anderson-Stuart theory.
\end{abstract}

(C) 2005 Elsevier B.V. All rights reserved.

Keywords: Glasses; Electrical conductivity; Ionic conduction; Anderson-Stuart model

\section{Introduction}

Since the discovery of fast ionic conductivity in glasses, it is believed that the diffusion of the mobile ions occurs via hopping motions between well-defined potential minima in the glassy network. In terms of practical applications, the germanate glasses are potential materials for low-loss optical devices such as optical fibers and IR transmitting windows, as also potential candidates for Raman fiber-optical amplifiers.

It is well known that the ionic conductivity increases rapidly when a network glassformer is modified by the addition of a metal alkali. Nevertheless, a generally accepted theory of ion transport in glass does not yet exist [1]. Several models have been proposed: they vary from thermodynamics with principles in models for liquid electrolytes, such as the weak electrolyte model [2], to models based on solid state concepts such as the jump diffusion model [3], the strong electrolyte model [4], and the dynamic structure model [5].

\footnotetext{
* Corresponding author.

E-mail address: pmlfn@ iris.ufscar.br (M.L.F. Nascimento).
}

\section{Theory}

Ionic conductivity $\sigma$ in glass is a thermally activated process of mobile ions by surmounting a potential barrier $E_{\mathrm{A}}$, of the form:

$\log \sigma=\log \sigma_{0}-0.434 E_{\mathrm{A}} / k_{\mathrm{B}} T$,

where $\sigma_{0}$ is the pre-exponential factor. Arrhenius plots of form $\log \sigma$ versus 1/T shown in Fig. 1 for 31 alkali germanate glasses demonstrate the noticeable scattering values of $E_{\mathrm{A}}$ against composition.

Extensive studies have recently been made for obtaining an 'universal' equation from the standpoint of glass structure. For example, Doi [6] presented conductivity values of 17 different glasses, not mentioned, that follows an 'universal' conductivity rule. Swenson and Börjesson [7] proposed a common cubic scaling relation of $\sigma$ with the expansion volumes of the networking forming units in salt-doped and -undoped glasses. This fact suggested that the glass network expansion, which is related to the available free volume, is a key parameter determining the increase of the high ionic conductivity in some types of fast ion conducting glasses. 


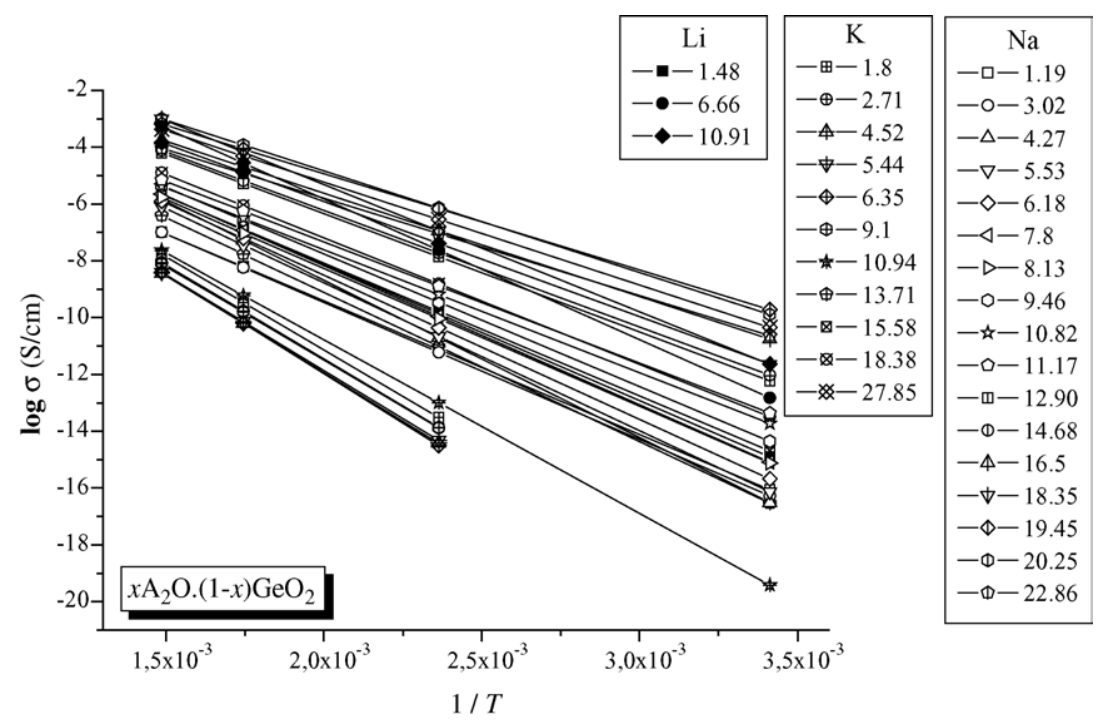

Fig. 1. Arrhenius plots of ionic conductivities in 31 binary alkali germanate glasses [15], of form $x \mathrm{~A}_{2} \mathrm{O} \cdot(1-x) \mathrm{GeO}_{2}(\mathrm{~A}=\mathrm{Li}, \mathrm{Na}, \mathrm{K}, x$ in mol\%, indicated). The temperatures measured are $20,150,300$ and $400^{\circ} \mathrm{C}$.

According to Adams and Swenson [8], the ion conduction should be determined by the ionic motion within an infinite pathway cluster. For various silver ion conducting glasses [9-10], it was found that the cubic root of the volume fraction $F$ of infinite pathways for a fixed valence mismatch threshold is closely related to both the absolute conductivity and the activation enthalpy of the conduction process:

$\log \sigma T \approx \sqrt[3]{F}=\log \sigma_{0}^{\prime}-0.434 E_{\mathrm{A}} / k_{\mathrm{B}} T$

where $\sigma_{0}^{\prime}$ is the pre-exponential factor (in $\mathrm{K} \mathrm{S} \mathrm{cm}^{-1}$ ).

\section{Results and discussion}

The activation enthalpy for ionic conduction, $E_{\mathrm{A}}$ was found to exhibit a maximum in some alkali germanate glasses at low alkali content. In few cases the composition dependence of $E_{\mathrm{A}}$ is in contrast with the corresponding variation in alkali silicates, where $E_{\mathrm{A}}$ decreases monotonically with increasing alkali content [11,12].

Concerning structure, the nature and distribution of sites hosting alkali metal ions in germanate glasses has been investigated by infrared reflectance spectroscopy as a function of alkali type and content. The analysis revealed the presence

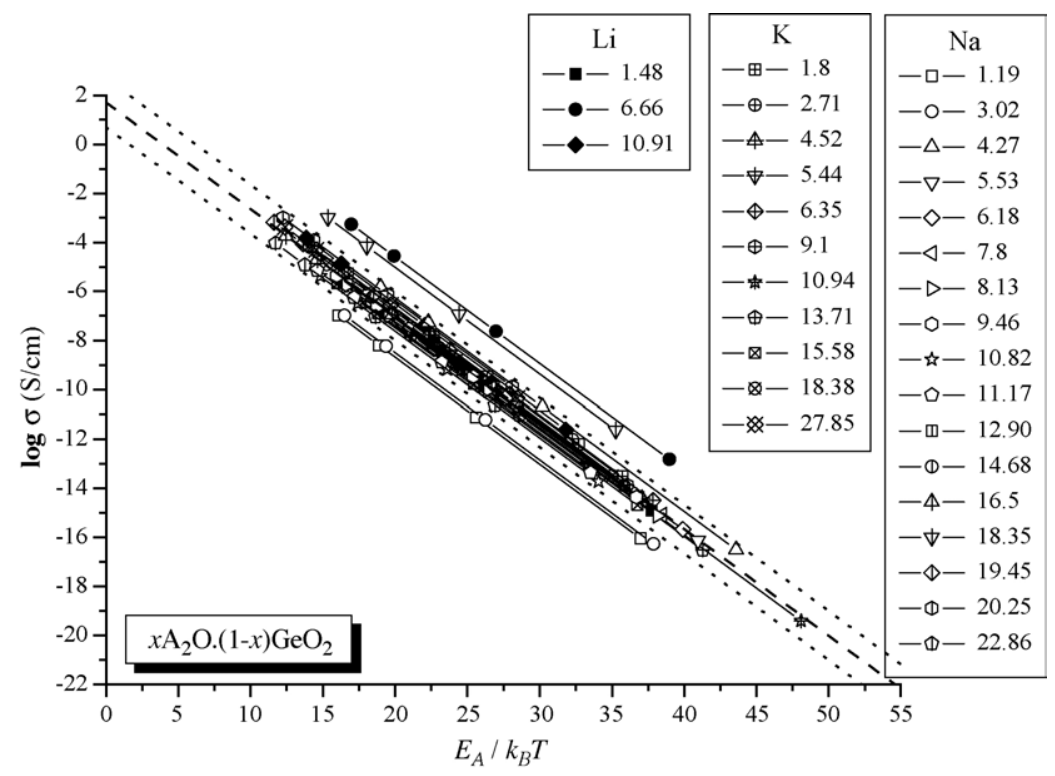

Fig. 2. Modified Arrhenius plots of ionic conductivities in 31 binary alkali germanate glasses [15], of form $x \mathrm{~A}_{2} \mathrm{O} \cdot(1-x) \mathrm{GeO} 2(\mathrm{~A}=\mathrm{Li}, \mathrm{Na}, \mathrm{K}, x$ in mol\%, indicated). The dashed line is the 'universal' curve, with $\sigma_{0}=50 \mathrm{~S} \mathrm{~cm}^{-1}$, and the dotted lines correspond to one order of magnitude higher or lower than Eq. (1). 
of two distributions of ionic sites at higher alkali content and just one distribution site at lower alkali content [13]. Roling et al. [13] suggested that the depolymerization of the network in alkali germanate, silicate and borate systems due to the formation of nonbridging oxygens leads to a strong decrease of the spatial extent of the ion hopping.

In a recent paper [14], the present authors have shown an 'universal' finding in binary borate glasses, considering both Eqs. (1) and (2). This short communication aims to present new results considering binary germanate glasses.

Fig. 2 shows modified Arrhenius plots of $\sigma$ for 31 binary alkali germanate glasses, of form $x \mathrm{~A}_{2} \mathrm{O} \cdot(1-x) \mathrm{GeO}_{2}(\mathrm{~A}=\mathrm{Li}$, $\mathrm{Na}, \mathrm{K}, x$ in mol\%, indicated [15]), ranging from $10^{-2} \mathrm{~S} \mathrm{~cm}^{-1}$ to less than $10^{-19} \mathrm{~S} \mathrm{~cm}^{-1}$ between 20 and $400^{\circ} \mathrm{C}$. The range of activation enthalpy $E_{\mathrm{A}}$ lie between 0.6 and $1.6 \mathrm{eV}$ in all glasses studied. These data were compared with the "universal' equation (Eq. (1)) using $\sigma_{0}=50 \mathrm{~S} \mathrm{~cm}^{-1}$. The "universal" equation, following Doy's sense, appears in Fig. 2 as a dashed line.

The substitution of the mobile ion by another kind of mobile ion introduces modification of the glass structure, as well as its ionic conductivity in various ways. Therefore, the results shown in Fig. 2 are remarkable in the sense that so many different binary alkali germanate glasses present linear curves of $\log \sigma$ versus $E_{\mathrm{A}} / k_{\mathrm{B}} T$ very close to each other and to the "universal curve".

This relationship between $\sigma$ and $E_{\mathrm{A}}$ values gives evidence for the validly of Eq. (1). Also one can conclude that the pre-exponential factor $\sigma_{0}$ varies only weakly with glass composition. There is a relationship between $\sigma$ and $E_{\mathrm{A}}$ values in a wide temperature range, i.e., $\sigma$ is strongly determined by $E_{\mathrm{A}} / k_{\mathrm{B}} T$ according to Eq. (1).

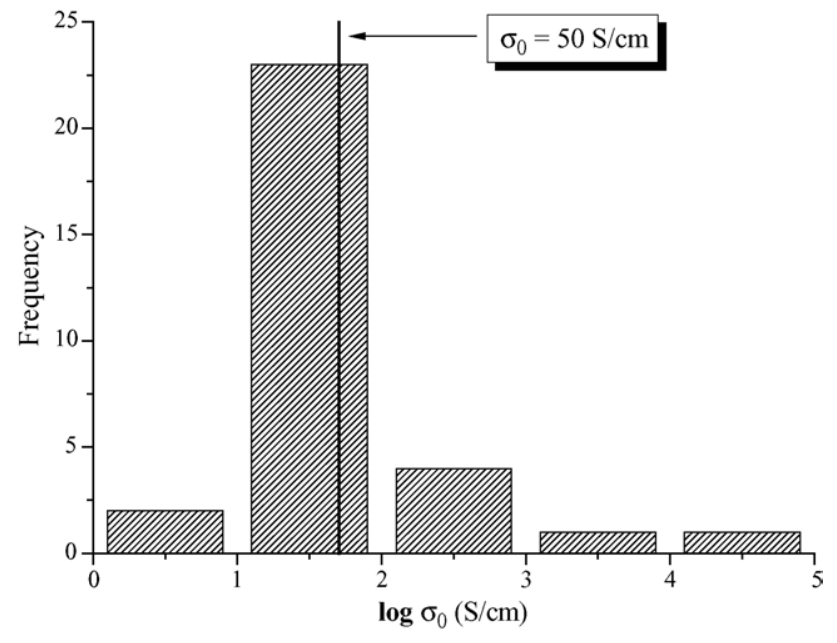

Fig. 3. Frequency distribution of $\sigma_{0}$ values from Eq. (1) corresponding to the experimental ionic conductivities of 31 binary alkali germanate glasses. The vertical line corresponds to $\sigma_{0}=50 \mathrm{~S} \mathrm{~cm}^{-1}$.

It is interesting to note that the increase in ionic conductivity with alkali content is almost entirely due to the fact that the activation enthalpy $E_{\mathrm{A}}$ required for a cation jump decreases, as presented in Ref. [4] considering silicate glasses. Thus, the $\sigma_{0}$-value in Eq. (1) is affected only weakly by alkali content.

It is important to note that the $\sigma$-values for several binary alkali borate glasses lie close to an "universal" curve. Although the $\sigma$-values for each glass at very low and very high temperatures differ by more than 17 orders of magnitude, for a given value of $E_{\mathrm{A}} / k_{\mathrm{B}} T$, the difference between large to small values of $\sigma$ is only one order of magnitude in $87 \%$ of the glass systems considered in Fig. 2. This means that, once obtained

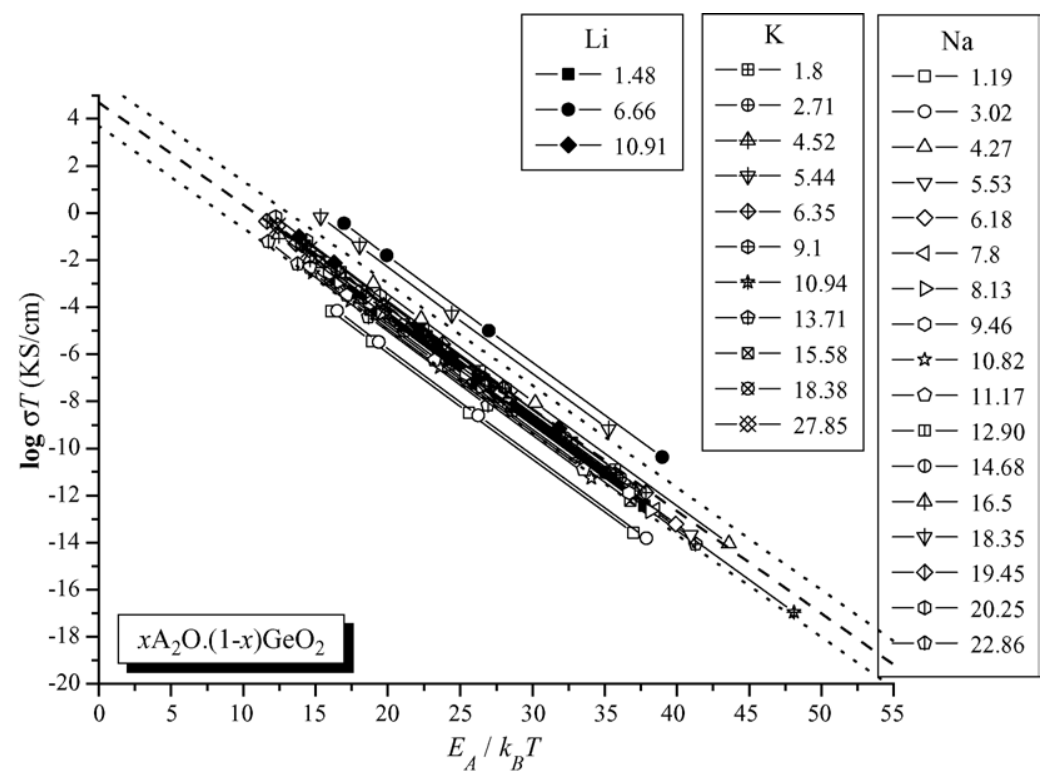

Fig. 4. Modified Arrhenius plots of ionic conductivities in 31 binary alkali germanate glasses [15], of form $x \mathrm{~A}_{2} \mathrm{O} \cdot(1-x) \mathrm{GeO} 2(\mathrm{~A}=\mathrm{Li}, \mathrm{Na}, \mathrm{K}, x$ in mol$\%$, indicated). The dashed line is the 'universal' curve, with $\sigma_{0}^{\prime}=50000 \mathrm{~K} \mathrm{~S} \mathrm{~cm}^{-1}$, and the dotted lines correspond to one order of magnitude higher or lower than Eq. (2). 
$\sigma$-value at a given temperature, the approximate value of $E_{\mathrm{A}}$ can be obtained from the "universal" equation, i.e., taking $\sigma_{0}=50 \mathrm{~S} \mathrm{~cm}^{-1}$ as the average value. The values of $\sigma$ for different temperatures can then be obtained using this $E_{\mathrm{A}}$-value. Of course, if $E_{\mathrm{A}}$-value is obtained by some other way, the ionic conductivity of a glass can be calculated using Eq. (1). The frequency of $\sigma_{0}$ distribution is shown in Fig. 3. It is possible to note that the medium value $\bar{\sigma}_{0}=54.0 \pm 5.9 \mathrm{~S} \mathrm{~cm}^{-1}$ is near $\sigma_{0}=50 \mathrm{~S} \mathrm{~cm}^{-1}$, the intercepting value at $y$-axis of Fig. 2 , considering 31 alkali germanate systems.

Another result, following Eq. (2), was obtained, and is presented in Fig. 4. The pre-exponential value was $\sigma_{0}^{\prime}=$ $50000 \mathrm{~K} \mathrm{~S} \mathrm{~cm}^{-1}$, considering the same conductivity data of Figs. 1 and 2. The conclusions for this case also follows the above described considering Eq. (1).

However, is not clear the reason for the decreasing tendency of the activation enthalpy with increasing alkali concentration. The expansion of the glass network and the introduction of the alkali ions into voids in the structure forming narrow pathways would lead to two effects that lower the activation enthalpy and thus promote the ionic conductivity. In terms of the Anderson-Stuart model [4], just two contributions were considered significant in $E_{\mathrm{A}}$ : (1) the bond energy of mobile cation, $E_{\mathrm{b}}$, that is the average energy that a cation requires to leave its site; (2) the elastic strain energy, $E_{\mathrm{s}}$, associated with the distortion of the glass network as the ion jumps from one equilibrium position to the next, that creates a "doorway" through which it can diffuse to a new site. The cation induced expansion of the network skeleton would lead to a lowering of the strain energy part $E_{\mathrm{s}}$ of the activation enthalpy and the formation of pathways, in which the cations may coordinate with oxygens of the network, leading to a lowering of $E_{\mathrm{b}}$. It is important to note that the Anderson-Stuart model confines to only one site distribution the hopping process.

\section{Conclusions}

In resume, there are strong connections between microscopic structure and ionic conductivity, fact based in the different types of composition in alkali germanate glasses presented. All these compositions fall into an identifiable pattern where conductivity is related to structure, in the form expressed by the "pathway volumes" of Adams and Swenson [16]. At first sight $E_{\mathrm{A}}$ and $k_{\mathrm{B}} T$ are independent, as described by Arrhenius plots, and $E_{\mathrm{A}}$ varies strongly with composition (the effect of glass composition is demonstrated clearly in Fig. 1). But modified Arrhenius plots showed that $E_{\mathrm{A}} / k_{\mathrm{B}} T$ is temperature and composition dependent and also related to $\sigma$. In other words, both $E_{\mathrm{A}}$ and $k_{\mathrm{B}} T$ (in the form $E_{\mathrm{A}} / k_{\mathrm{B}} T$ ) are related to the ' $F$ ' factor of Adams and Swenson. Furthermore, frequency distribution values of pre-exponential $\sigma_{0}$ showed an average result of $\bar{\sigma}_{0}=54.0 \pm 5.9 \mathrm{~S} \mathrm{~cm}^{-1}$. It should be necessary to emphasize that the findings shown in Figs. 2 and 4 demonstrate that modified Arrhenius plots (Eqs. (1) and (2)) are valid. Further studies on other binary oxide glassformers as presented in Figs. 2 and 4 will refuse or recognize this 'universal' finding.

\section{Acknowledgements}

The Brazilian agencies CAPES, CNPq and FAPESP are acknowledged for their financial support. Special thanks to Prof. Dr. Vladimir M. Fokin (Vavilov State Optical Institute, Russia) and his valuable discussions.

\section{References}

[1] C.A. Angell, Annu. Rev. Phys. Chem. 43 (1992) 693.

[2] D. Ravaine, J.L. Souquet, Phys. Chem. Glasses 18 (1977) 27.

[3] K. Funke, Prog. Solid State Chem. 22 (1993) 11.

[4] O.L. Anderson, D.A. Stuart, J. Am. Ceram. Soc. 37 (1954) 573.

[5] A. Bunde, M.D. Ingram, P. Maass, J. Non-Cryst. Solids 172-174 (1994) 1222

[6] A. Doi, J. Mater. Sci. 39 (2004) 6827.

[7] J. Swenson, L. Börjesson, Phys. Rev. Lett. 77 (1996) 3569.

[8] S. Adams, J. Swenson, Phys. Chem. Chem. Phys. 4 (2002) 3179.

[9] J. Swenson, S. Adams, Phys. Rev. B 64 (2002) 024204.

[10] S. Adams, J. Swenson, Solid State Ionics 154-155 (2002) 151.

[11] E.I. Kamitsos, Y.D. Yannopoulos, H. Jain, W.C. Huang, Phys. Rev. B 54 (1996) 9775.

[12] H. Jain, W.C. Huang, E.I. Kamitsos, Y.D. Yannopoulos, J. Non-Cryst. Solids 222 (1997) 361.

[13] B. Roling, C. Martiny, S. Brückner, Phys. Rev. B 63 (2001) 214203.

[14] M.L.F. Nascimento, S. Watanabe, J. Mater. Sci., in press.

[15] Sciglass ${ }^{\circledR}$ Database. http://www.esm-software.com/sciglass

[16] S. Adams, J. Swenson, Phys. Rev. Lett. 84 (2000) 4144. 